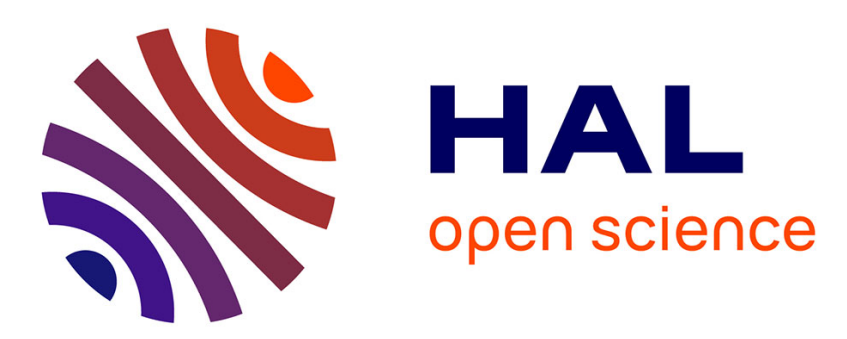

\title{
Characterization of the evolution of the train dynamic response under the effect of track irregularities
}

Nicolas Lestoille, Christian Soize, Christine Funfschilling

\section{To cite this version:}

Nicolas Lestoille, Christian Soize, Christine Funfschilling. Characterization of the evolution of the train dynamic response under the effect of track irregularities. 24th International Symposium on Dynamics of Vehicles on Roads and Tracks (IAVSD 2015), Aug 2015, Graz, Austria. pp.1-7. hal-01192562

\section{HAL Id: hal-01192562 \\ https://hal.science/hal-01192562}

Submitted on 2 Sep 2015

HAL is a multi-disciplinary open access archive for the deposit and dissemination of scientific research documents, whether they are published or not. The documents may come from teaching and research institutions in France or abroad, or from public or private research centers.
L'archive ouverte pluridisciplinaire HAL, est destinée au dépôt et à la diffusion de documents scientifiques de niveau recherche, publiés ou non, émanant des établissements d'enseignement et de recherche français ou étrangers, des laboratoires publics ou privés. 


\title{
Characterization of the evolution of the train dynamic response under the effect of track irregularities
}

\author{
N. Lestoille \\ SNCF, Innovation and Research Department, Paris, France \\ Université Paris-Est, Modélisation et Simulation Multi-Échelle (MSME UMR 8208 CNRS), \\ Marne-la-Vallée, France \\ C. Soize \\ Université Paris-Est, Modélisation et Simulation Multi-Échelle (MSME UMR 8208 CNRS), \\ Marne-la-Vallée, France \\ C. Funfschilling \\ SNCF, Innovation and Research Department, Paris, France
}

\begin{abstract}
There is a great interest to predict the long-time evolution of the track irregularities for a given track portion of the high-speed train network, in order to be able to anticipate the start off of the maintenance operations. In this paper, a stochastic predictive model is proposed for predicting the long-time evolution of a vector-valued random dynamic indicator related to the nonlinear dynamic responses of the high-speed train excited by the stochastic track irregularities. The long-time evolution of the vector-valued random indicator is modeled by a discrete non-Gaussian nonstationary stochastic model (ARMA type model), for which the coefficients are time-dependent. The quality assessment of the stochastic predictive model is presented, which validates the proposed stochastic model.
\end{abstract}

\section{INTRODUCTION}

The maintenance work on railways tracks is very costly for the railways companies which are permanently seeking to improve the maintenance strategy. In particular, the prediction of the long-time evolution of the high-speed train dynamics and of the track geometry would help railways companies to anticipate their maintenance operations and to improve their planning. Several approaches for improving the track maintenance strategy have been studied in Andrews et al. (2014); Chaolong et al. (2012); Mizuno et al. (2008). In this work, the long-time evolution of a given track portion is characterized by the evolution of a vector-valued random dynamic indicator related to the train dynamic responses. The goal is to build and to identify a stochastic predictive model of long-time evolution of this vector-valued random dynamic indicator by using experimental data generated at discrete time $\left\{\tau_{1}, \tau_{2}, \ldots, \tau_{K}\right\}$, and then to predict the statistics of the vector-valued random dynamic indicator at long time $\tau_{K+1}$.

Section 2 deals with the construction of the random dynamic indicator, using a stochastic modeling of the track irregularities of the given track portion and the simulation of the train dynamic response on the track portion. Then, the construction of the stochastic predictive model of the random dynamic indicator is presented in Section 3 . In Section 4 the prediction of the random indicator is computed. 


\section{STOCHASTIC MODELING OF THE TRAIN DYNAMIC RESPONSE}

\subsection{Local stochastic modeling of the track irregularities}

For the given track portion and for discrete long times $\tau_{k}, k=1, \ldots, K$, the track irregularities are measured by a measuring train ( $K=12$ measures are available for the given track portion). The spatial sampling of the four track irregularities around their mean values (the track design) is measured at $N_{s}+1$ sampling points of the curvilinear abscissa of the track portion and the measures at $\tau_{k}$ are denoted by $\mathbf{x}_{\tau_{k}}^{\text {meas }}=\left(\mathbf{x}_{\tau_{k}}^{\text {meas, }, 1}, \mathbf{x}_{\tau_{k}}^{\text {meas, } 2}, \mathbf{x}_{\tau_{k}}^{\text {meas, } 3}, \mathbf{x}_{\tau_{k}}^{\text {meas }, 4}\right)$ in $\mathbb{R}^{4\left(N_{s}+1\right)}$. The track irregularities vector discretizing the random field is modeled by a non-Gaussian centered random variable $\widetilde{\mathbf{X}}_{\tau_{k}}=\left(\widetilde{\mathbf{X}}_{\tau_{k}}^{1}, \widetilde{\mathbf{X}}_{\tau_{k}}^{2}, \widetilde{\mathbf{X}}_{\tau_{k}}^{3}, \widetilde{\mathbf{X}}_{\tau_{k}}^{4}\right)$ with values in $\mathbb{R}^{4\left(N_{s}+1\right)}$. As explained in Lestoille et al. (2014, 2015) and on the base of the works published in Perrin et al. (2012, 2013, 2015), this local stochastic modeling of the track irregularities vector is constructed using the experimental measurements $\mathbf{x}_{\tau_{k}}^{\text {meas }}$ with $k=1, \ldots, K$ and is written as

$$
\widetilde{\mathbf{X}}_{\tau_{k}}^{\kappa}=\left[Q^{\kappa}\right]\left(\boldsymbol{\eta}_{\tau_{k}}^{\text {meas }}+\delta_{\tau_{1}}^{\kappa, \text { opt }} \mathbf{G}^{\kappa}\right), \quad \kappa=1,2,3,4, \quad k=1, \ldots, K
$$

in which

- $\left[Q^{\kappa}\right]$ is a rectangular $\left(\left(N_{s}+1\right) \times N_{\eta}\right)$ real matrix and is an extraction from the orthogonal $\left(4\left(N_{s}+1\right) \times N_{\eta}\right)$ matrix $[Q]$ that contains the vectors of the global stochastic modeling of the track geometry developed in Perrin et al. (2012, 2013, 2015).

- $\boldsymbol{\eta}_{\tau_{k}}^{\text {meas }}$ defined on $\mathbb{R}^{N_{\eta}}$ is the projection of the measurement $\mathbf{x}_{\tau_{k}}^{\text {meas }}$ on the basis of the global stochastic modeling of the track irregularities.

- $\boldsymbol{\delta}_{\tau_{1}}^{\text {opt }}=\left(\delta_{\tau_{1}}^{1, \text { opt }}, \delta_{\tau_{1}}^{2 \text { opt }}, \delta_{\tau_{1}}^{3 \text { opt }}, \delta_{\tau_{1}}^{4 \text {,opt }}\right)$ is the optimal value of hyperparameter $\boldsymbol{\delta}_{\tau_{1}}$, which is identified by using the maximum log-likelihood method and the experimental measurements of the track iregularities, as explained in Lestoille et al. (2015). For this track portion, the optimal value is $\boldsymbol{\delta}_{\tau_{1}}^{\text {opt }}=(0.15,0.9,0.85,0.8)$.

- $\mathbf{G}=\left(\mathbf{G}^{1}, \mathbf{G}^{2}, \mathbf{G}^{3}, \mathbf{G}^{4}\right)$ is a Gaussian second-order centered random vector defined on the probability space $(\Theta, \mathcal{F}, \mathcal{P})$ with values in $\mathbb{R}^{4 N_{\eta}}$, for which its covariance matrix is the identity matrix. This random vector allows to adapt the global stochastic model to the local one for the given track portion.

\subsection{Constructing the vector-valued random dynamic indicator}

The vector-valued indicator is defined in order to characterize the high-speed train dynamic response on a given track portion of length $S$. It is based on criteria related to the high-speed train dynamic response that are described in norm UIC 518 UIC (2009) for the certification of railway vehicles and denoted by $\mathbf{C}$ with values in $\mathbb{R}^{N}$. The number of components of the vector-valued indicator is $N=9$. For $j=1, \ldots, 9$, these components are

- $C_{1}$ : the lateral acceleration of the first bogie in the train.

- $C_{2}$ : the vertical acceleration of the first bogie in the train.

- $C_{3}$ : the lateral acceleration of the third bogie in the train.

- $C_{4}$ : the lateral acceleration of the second coach in the train.

- $C_{5}$ : the sum of lateral forces on the ninth wheelset in the train.

- $C_{6}$ : the sum of vertical forces on the first wheelset in the train.

- $C_{7}$ : the sum of vertical forces of the second wheelset in the train.

- $C_{8}$ : the sum of vertical forces of the tenth wheelset in the train.

- $C_{9}$ : the difference between right-wheel and left-wheel vertical forces of the tenth wheelset in the train.

The vector-valued random dynamic indicator, denoted by $\mathbf{C}^{\text {mod }}$, is constructed by using a train nonlinear dynamic computational model for which model uncertainties are taken into account in introducing a non-Gaussian noise $\mathbf{B}^{\text {out }}$ defined on the probability space $\left(\Theta^{\prime}, \mathcal{F}^{\prime}, \mathcal{P}^{\prime}\right)$, which is statistically independent of random vector $\mathbf{G}$ (see hereinafter). The stochastic solver used is the Monte-Carlo method which is performed with $\nu=2000$ independent realizations of the stochastic model. The steps achieved to evaluate the random dynamic indicator are the following:

- $\nu=2000$ independent realizations $\mathbf{G}\left(\theta_{1}\right), \ldots, \mathbf{G}\left(\theta_{\nu}\right)$ of $\mathbf{G}=\left(\mathbf{G}^{1}, \mathbf{G}^{2}, \mathbf{G}^{3}, \mathbf{G}^{4}\right)$ are generated. 
- These $\nu$ independent realizations of $\mathbf{G}$ are used for constructing, for each $k=1, \ldots, K$, the $\nu$ independent realizations of random vector $\widetilde{\mathbf{X}}_{\tau_{k}}=\left(\widetilde{\mathbf{X}}_{\tau_{k}}^{1}, \widetilde{\mathbf{X}}_{\tau_{k}}^{2}, \widetilde{\mathbf{X}}_{\tau_{k}}^{3}, \widetilde{\mathbf{X}}_{\tau_{k}}^{4}\right)$ by using Eq. (1). For each realization of the track irregularities $\widetilde{\mathbf{X}}_{\tau_{k}}$, the deterministic train dynamic response induced by this realization of the track irregularities is computed with a multibody commercial software (Vampire), and the $\nu$ corresponding independent realizations $\mathbf{C}^{\operatorname{sim}}\left(\tau_{k} ; \theta_{1}\right), \ldots \mathbf{C}^{\operatorname{sim}}\left(\tau_{k} ; \theta_{\nu}\right)$ of the random vector-valued dynamic indicator $\mathbf{C}^{\operatorname{sim}}\left(\tau_{k}\right)$ are computed.

- Then, $\nu$ independent realizations of the $\mathbb{R}^{N}$-valued non-Gaussian second-order random vector $\mathbf{B}^{\text {out }}=\left(B_{1}^{\text {out }}, \ldots, B_{N}^{\text {out }}\right)$ are generated. Random vector $\mathbf{B}^{\text {out }}$ has been beforehand identified in introducing its polynomial chaos representation (see for instance Ghanem and Spanos (2003); Le Maitre and Knio (2010)) and in identifying its coefficients by using the maximum-likelihood method and the experimental measurements of the train dynamic response Soize (2012).

- The $\nu$ corresponding independent realizations of the family $\mathcal{C}^{\text {mod }}$ of the vector-valued random dynamic indicators $\left\{\mathbf{C}^{\bmod }\left(\tau_{1}\right), \ldots, \mathbf{C}^{\bmod }\left(\tau_{K}\right)\right\}$, denoted by $\mathcal{C}^{\bmod }\left(\theta_{1}, \theta_{1}^{\prime}\right), \ldots, \mathcal{C}^{\bmod }\left(\theta_{\nu}, \theta_{\nu}^{\prime}\right)$ and defined on the product of the probability spaces $(\Theta, \mathcal{F}, \mathcal{P})$ and $\left(\Theta^{\prime}, \mathcal{F}^{\prime}, \mathcal{P}^{\prime}\right)$, are computed such as

$$
\begin{aligned}
& C_{j}^{\text {mod }}\left(\tau_{k} ; \theta_{\ell}, \theta_{\ell}^{\prime}\right)=C_{j}^{\operatorname{sim}}\left(\tau_{k} ; \theta_{\ell}\right) \exp \left(B_{j}^{\text {out }}\left(\theta_{\ell}^{\prime}\right)\right), \\
& j=1, \ldots, N \quad, \quad k=1, \ldots, K \quad, \quad \ell=1, \ldots, \nu .
\end{aligned}
$$

\section{STOCHASTIC MODELING OF THE LONG-TIME EVOLUTION OF THE VECTOR-VALUED RANDOM INDICATOR}

\subsection{Choosing the stochastic predictive model}

Using the statistics of the vector-valued random dynamic indicator $\mathbf{C}^{\bmod }\left(\tau_{1}\right), \ldots, \mathbf{C}^{\bmod }\left(\tau_{K}\right)$, which have been calculated for all long time $\tau_{1}, \ldots, \tau_{K}$ in Section 2, a stochastic predictive model of the vector-valued random dynamic indicator to long-time evolution of track irregularities is constructed and then identified. The vector-valued random dynamic indicator that is predicted by the stochastic predictive model, is represented by the $\mathbb{R}^{N}$-valued time series $\mathbf{C}^{k}$ (also denoted by $\mathbf{C}\left(\tau_{k}\right)$ ). The initial condition of the model is set as $\mathbf{C}^{1}=\mathbf{C}^{\bmod }\left(\tau_{1}\right)$. If the stochastic predictive model were perfect (no error), we would have $\mathbf{C}^{\bmod }\left(\tau_{k}\right)=\mathbf{C}\left(\tau_{k}\right)$ for $k=1, \ldots, K$ (which is not the case). Using the stochastic predictive model, the statistics of the vector-valued random dynamic indicator $\mathbf{C}^{K+1}$ are calculated for discrete long time $\tau_{K+1}$. Such a prediction is obtained using the identified stochastic predictive model starting at initial condition $\mathbf{C}^{K}=\mathbf{C}^{\bmod }\left(\tau_{K}\right)$. The construction and the identification of the stochastic predictive model of the vector-valued random dynamic indicator are relatively difficult for the following reasons:

- The long-time evolution is strongly nonstationary (and consequently statistics cannot be enriched by using time averaging estimators).

- The experimental data are very limited because only one measurement of the track geometry of the given portion is available at each discrete long time $\tau_{k}$.

- The value $K$ of the number of discrete long times used for predicting the statistics of the vectorvalued random dynamic indicator at discrete long time $\tau_{K+1}$ is very low (typically $K$ is of order 10).

- The vector-valued random dynamic indicator is a non-Gaussian random vector, in particular the initial value $\mathbf{C}^{\bmod }\left(\tau_{1}\right)$ is non-Gaussian, which means that the time series $\mathbf{C}^{\bmod }\left(\tau_{1}\right), \ldots$, $\mathbf{C}^{\bmod }\left(\tau_{K}\right)$ is non-Gaussian and nonstationary.

Two steps are required for predicting the statistics of vector-valued dynamic indicator at long time. The first one is related to the choice and the construction of a stochastic predictive model and the second one consists in identifying it by solving a statistical inverse problem. The time series $\mathbf{C}\left(\tau_{1}\right), \ldots, \mathbf{C}\left(\tau_{K}\right)$ for which the discrete time evolution stochastic model has to be constructed using time series data $\mathbf{C}^{\bmod }\left(\tau_{1}\right), \ldots, \mathbf{C}^{\bmod }\left(\tau_{K}\right)$ is nonstationary, and consequently the identification requires to solve a nonstationary statistical inverse problem. A general method for such a problem consists in using the Bayesian filtering and, in our case, by using a discrete time evolution model. 
Since the vector-valued dynamic indicator is nonstationary and non-Gaussian, the method adopted for constructing and identifying the stochastic predictive model consists in using a nonstationary ARMA model Priestley (1981, 1988); Hamilton (1994) with a non-Gaussian random initial condition. The proposed stochastic predictive model is a particular nonstationary nonGaussian one-order Markov chain which is written as

$$
\mathbf{C}^{k}=\left(\left[I_{N}\right]-\Delta \tau_{k}[A]\right) \mathbf{C}^{k-1}+\Delta \tau_{k} \mathbf{g}^{k}+\left[h^{k}\right] \Delta \mathbf{W}^{k} \quad, \quad k=2, \ldots, K,
$$

with the non-Gaussian random initial condition

$$
\mathbf{C}^{1}=\mathbf{C}^{\bmod }\left(\tau_{1}\right)
$$

in which

- $\left[I_{N}\right]$ is the identity matrix in $\mathbb{R}^{N \times N}$.

- $[A]$ is a matrix in $\mathbb{R}^{N \times N}$, which has to be identified.

- $\Delta \tau_{k}=\tau_{k}-\tau_{k-1}$ are given time steps that depend on $k$.

- $\Delta \mathbf{W}^{k}=\sqrt{\Delta \tau_{k}} \mathcal{N}^{k}$, in which $\mathcal{N}^{2}, \ldots, \mathcal{N}^{K}$ are independent Gaussian normalized random vectors defined on a third probability space $\left(\Theta^{\prime \prime}, \mathcal{F}^{\prime \prime}, \mathcal{P}^{\prime \prime}\right)$, with values in $\mathbb{R}^{N}\left(E\left\{\mathcal{N}^{k}\right\}=\right.$ $\left.0, E\left\{\mathcal{N}^{k}\left(\mathcal{N}^{k}\right)^{T}\right\}=\left[I_{N}\right]\right)$. Therefore, $E\left\{\Delta \mathbf{W}^{k} \otimes \Delta \mathbf{W}^{k}\right\}=\Delta \tau_{k}\left[I_{N}\right]$. The family of random vectors $\left\{\mathcal{N}^{k}, k \geq 2\right\}$ is statistically independent of random vectors $\mathbf{G}$ and $\mathbf{B}^{\text {out }}$, and consequently, is independent of $\mathbf{C}^{\bmod }\left(\tau_{1}\right)$.

- $\{\mathbf{g}\}=\left\{\mathbf{g}^{2}, \ldots, \mathbf{g}^{K}\right\}$ is a family of $K-1$ vectors in $\mathbb{R}^{N}$, which has to be identified.

- $\{[h]\}=\left\{\left[h^{2}\right], \ldots,\left[h^{K}\right]\right\}$ is a family of $(K-1)$ real matrices in $\mathbb{R}^{N \times N}$, which has to be identified. For all $k,\left[h^{k}\right]$ is chosen as a lower triangular matrix with positive diagonal entries.

- in the initial condition, $\mathbf{C}^{\bmod }\left(\tau_{1}\right)$ is a non-Gaussian second-order $\mathbb{R}^{N}$-valued random variable whose probability distribution is known (estimated using the stochastic computational model of the high-speed train dynamics described in Section 2).

The nonstationarity property is induced by the coefficients $\mathbf{g}^{k}$ and $\left[h^{k}\right]$ that depend on discrete time $\tau_{k}$, represented by index $k$. The matrix $[A]$ is chosen independent on discrete time $k$. It should be noticed that, if matrix $[A]$ had been chosen as a function of $k$, the time series $\left[A^{k}\right], \mathbf{g}^{k}$, and $\left[h^{k}\right]$ could not be identified by the loss of data. Moreover, if the initial condition $\mathbf{C}^{1}=\mathbf{C}^{\bmod }\left(\tau_{1}\right)$ were a deterministic vector or a Gaussian random vector, then the nonstationary time series $\mathbf{C}\left(\tau_{k}\right)$ would be Gaussian. Nevertheless, since random vector $\mathbf{C}^{\bmod }\left(\tau_{1}\right)$ is not Gaussian, time series $\mathbf{C}\left(\tau_{k}\right)$ generated by the predictive model above is a non-Gaussian nonstationary time series.

\subsection{Modeling the long time evolution of vector-valued random dynamic indicator}

With such a proposed stochastic predictive model, the opitmal values $\left\{\left[A^{\mathrm{opt}}\right],\left\{\mathbf{g}^{\mathrm{opt}}\right\},\left\{\left[h^{\mathrm{opt}}\right]\right\}\right\}$ of the parameters $\{[A],\{\mathbf{g}\},\{[h]\}\}$ have to be identified using data $\mathbf{C}^{\bmod }\left(\tau_{1}\right), \ldots, \mathbf{C}^{\bmod }\left(\tau_{K}\right)$, for which the statistics have been estimated in Section 2. The identification is done using the classical least-squares method with weights, for which the cost function is constructed using the first- and second-order moments equations associated with Eq. (3). For each $k=1, \ldots, K$, the vector-valued random dynamic indicator $\mathbf{C}^{k}$ is constructed using Eq. (3), with the initial condition $\mathbf{C}^{1}=\mathbf{C}^{\text {mod }}\left(\tau_{1}\right)$, using the optimal parameters $\left\{\left[A^{\text {opt }}\right],\left\{\mathbf{g}^{\text {opt }}\right\},\left\{\left[h^{\text {opt }}\right]\right\}\right\}$.

In the following, for $k=2, \ldots, K$, we present a comparison of the vector-valued dynamic indicator $\mathbf{C}^{\bmod }\left(\tau_{k}\right)$, constructed in Section 2 , with the modeled dynamic indicator $\mathbf{C}^{k}$. In order to limit the number of figures, we restrict the presentation to the component $j=6$ of the vectorvalued dynamic indicator. The results for the other components are similar. For each quantity, $C_{6}^{\bmod }\left(\tau_{k}\right)$ and $C_{6}^{k}$, denoted hereinafter as $D\left(\tau_{k}\right)$, Figure 1 displays the graph $k \mapsto d^{i}\left(\tau_{k}\right)$ in which $d^{i}\left(\tau_{k}\right)$ is such that $\operatorname{Prob}\left\{D\left(\tau_{k}\right) \leq d^{i}\left(\tau_{k}\right)\right\} \geq q^{i}$, in which $q^{i}$ is the quantile varying in the interval $[0.4,0.98]$. This figure shows that the stochastic model of the long-time evolution defined by Eqs. (3) and (4) is a good approximation for representing the long-time evolution of the vector-valued dynamic indicator. 


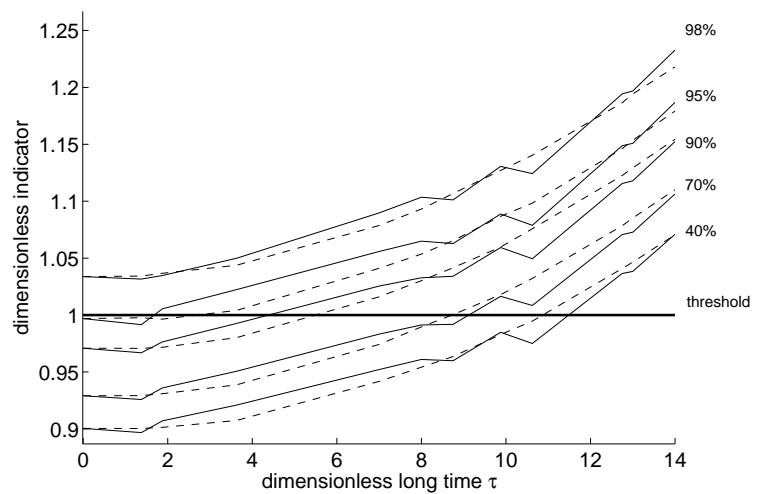

Figure 1. Quantiles of the dimensionless long-time evolution for the dimensionless indicator $D\left(\tau_{k}\right)=$ $C_{6}^{\text {mod }}\left(\tau_{k}\right)$ (solid line) and $D\left(\tau_{k}\right)=C_{6}^{k}$ (dashed line), graphs $k \mapsto d^{i}\left(\tau_{k}\right)$, in which $d^{i}\left(\tau_{k}\right)$ is such that $\operatorname{Prob}\left\{D\left(\tau_{k}\right) \leq d^{i}\left(\tau_{k}\right)\right\} \geq q^{i}$ for different values in percent of the quantile $q^{i}$ belonging to the interval $[40 \%, 98 \%]$. The horizontal line corresponds to the threshold level $c_{6}^{*}$.

\section{PREDICTION OF THE VECTOR-VALUED RANDOM INDICATOR $\mathbf{C}^{K+1}$}

Once the stochastic predictive model is identified, this model can be used for predicting the statistics of the non-Gaussian random vector $\mathbf{C}^{K+1}$. However, the values of $\mathbf{g}^{K+1}$ and $\left[h^{K+1}\right]$ are unknown. We then propose to represent $\mathbf{g}^{k}$ and $\left[h^{k}\right]$ as the values $\mathbf{g}^{\text {aff }}\left(\tau_{k}\right)$ and $\left[h^{\text {aff }}\left(\tau_{k}\right)\right]$ at $\tau_{k}$ of affine functions $\mathbf{g}^{\text {aff }}$ and $\left[h^{\text {aff }}\right]$ (a more higher-degree representations could be introduced, but it has been seen that no gain can be obtained with respect to the one-degree (affine) representation). The optimal values for the parameters of the affine functions are computed solving optimization problems with a classical least-squares method. The prediction $\mathbf{C}^{\text {aff, } K+1}$ of the vector-valued random indicator $\mathbf{C}^{K+1}=\mathbf{C}\left(\tau_{K+1}\right)$ at long time $\tau_{K+1}$, given $\mathbf{C}^{\bmod }\left(\tau_{K}\right)$, is then estimated using Eq. (3) that yields

$$
\begin{aligned}
\mathbf{C}^{\text {aff }, K+1}=\left(\left[I_{N}\right]-\Delta \tau_{K+1}\left[A^{\mathrm{opt}}\right]\right) \mathbf{C}^{\bmod }\left(\tau_{K}\right)+\Delta \tau_{K+1} \mathbf{g}^{\mathrm{aff}}\left(\tau_{K+1}\right) & \\
& +\left[h^{\mathrm{aff}}\left(\tau_{K+1}\right)\right] \Delta \mathbf{W}^{K+1} .
\end{aligned}
$$

The prediction at $\tau_{K+1}$ of the quantiles of the dimensionless long-time evolution for the dimensionless indicator $D\left(\tau_{K+1}\right)=C_{6}^{\text {aff, } K+1}$ given $D\left(\tau_{k}\right)=C_{6}^{\bmod }\left(\tau_{k}\right), k=1, \ldots, K$, is represented in Figure 2, where graphs $k \mapsto d^{i}\left(\tau_{k}\right)$, in which $d^{i}\left(\tau_{k}\right)$ is such that $\operatorname{Prob}\left\{D\left(\tau_{k}\right) \leq d^{i}\left(\tau_{k}\right)\right\} \geq$ $q^{i}$ for different values in percent of the quantile $q^{i}$ belonging to the interval $[40 \%, 98 \%]$, are displayed.

\section{QUALITY ASSESSMENT OF THE STOCHASTIC PREDICTIVE MODEL}

The relevance of the stochastic predictive model can be obtained by quality assessment, in which the predictions $\mathbf{C}^{\text {aff, } K-1}$ and $\mathbf{C}^{\text {aff, } K}$ are performed given the known random vector $\mathbf{C}^{\text {mod }}\left(\tau_{K-2}\right)$. This prediction is carried out using Eq. (5) that is rewritten as

$$
\begin{aligned}
\mathbf{C}^{\mathrm{aff}, k}=\left(\left[I_{N}\right]-\Delta \tau_{k}\left[A^{\mathrm{opt}}\right]\right) \mathbf{C}^{\mathrm{aff}, k-1}+\Delta \tau_{k} \mathbf{g}^{\mathrm{aff}}\left(\tau_{k}\right) & \\
& +\left[h^{\mathrm{aff}}\left(\tau_{k}\right)\right] \Delta \mathbf{W}^{k} \quad, \quad k=K-1, K,
\end{aligned}
$$

with the initial condition

$$
\mathbf{C}^{\text {aff }, K-2}=\mathbf{C}^{\text {mod }}\left(\tau_{K-2}\right) \text {. }
$$

The quality assessment is then evaluated in comparing $\mathbf{C}^{\text {aff, } K-1}$ with $\mathbf{C}^{\bmod }\left(\tau_{K-1}\right)$, and then in comparing $\mathbf{C}^{\text {aff }, K}$ with $\mathbf{C}^{\bmod }\left(\tau_{K}\right)$. 


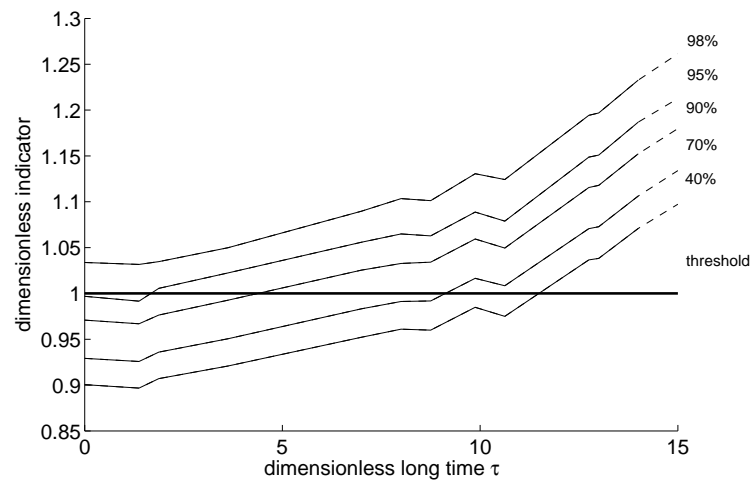

Figure 2. Quantiles of the dimensionless long-time evolution for the dimensionless indicator $D\left(\tau_{k}\right)=$ $C_{6}^{\text {mod }}\left(\tau_{k}\right)$ (solid line) and $D\left(\tau_{K+1}\right)=C_{6}^{\text {aff, } K+1}$ (dashed line), graphs $k \mapsto d^{i}\left(\tau_{k}\right)$, in which $d^{i}\left(\tau_{k}\right)$ is such that $\operatorname{Prob}\left\{D\left(\tau_{k}\right) \leq d^{i}\left(\tau_{k}\right)\right\} \geq q^{i}$ for different values in percent of the quantile $q^{i}$ belonging to the interval $[40 \%, 98 \%]$. The horizontal line corresponds to the threshold level $c_{6}^{*}$.

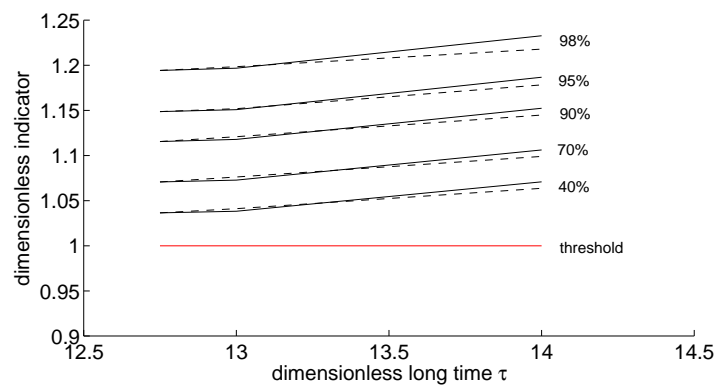

Figure 3. Quality assessment of the stochastic predictive model comparing the quantiles of $C_{6}^{\text {aff, } k}$ (dashed line) with the quantiles of $C_{6}^{\bmod }\left(\tau_{k}\right)$ (solid line) for $k=K-1$ and for $k=K$.

For the quantiles of the dimensionless indicator $D\left(\tau_{k}\right)=C_{6}^{\bmod }\left(\tau_{k}\right)$ and $D\left(\tau_{k}\right)=C_{6}^{\text {aff, }, k}$ for $k=K-1$ and for $k=K$, the quality assessment can be viewed in Figure 3 that displays the graphs $k \mapsto d^{i}\left(\tau_{k}\right)$, in which $d^{i}\left(\tau_{k}\right)$ is such that $\operatorname{Prob}\left\{D\left(\tau_{k}\right) \leq d^{i}\left(\tau_{k}\right)\right\} \geq q^{i}$ for different values in percent of the quantile $q^{i}$ belonging to the interval $[40 \%, 98 \%]$. This result shows that the stochastic predictive model is good enough.

\section{CONCLUSION}

For the companies in charge of the maintenance of railways networks, there is a great interest to predict the long-time evolution of the track irregularities for a given track portion of the network, in order to be able to anticipate the start off of the maintenance operations. In this work, the long-time evolution of the track irregularities of a given track portion has been evaluated through a vector-valued random indicator related to the train dynamic response induced by the random track irregularities. The long-time evolution of this vector-valued random indicator is modeled by a discrete non-Gaussian nonstationary stochastic model (ARMA type model), for which the coefficients are time-dependent. These coefficients have been identified by a least-squares method and fitted on long time, using experimental measurements.

The proposed stochastic predictive model, based on big data made up of a lot of experimental measurements performed for the french high-speed train network, allows for predicting the statistical quantities of the vector-valued random dynamic indicator for long times for which no measurements have been performed yet. It has been demonstrated that this proposed stochastic predictive model is good enough. This proposed model can help to determine the best time to start off the maintenance operations as a function of a chosen threshold for the indicator. 


\section{REFERENCES}

Andrews, J., D. Prescott, and F. D. Rozières 2014. A stochastic model for railway track asset management. Reliability Engineering and System Safety, 130:76-84.

Chaolong, J., X. Weixiang, W. Futian, and W. Hanning 2012. Track Irregularity Time Series Analysis and Trend Forecasting. Discrete Dynamics in Nature and Society, 2012.

Ghanem, R. and P. D. Spanos 2003. Stochastic Finite Elements: A Spectral Approach, rev. ed. New York: Dover Publications, New York.

Hamilton, J. D. 1994. Time Series Analysis. Princeton, USA: Princeton University Press.

Le Maitre, O. and O. Knio 2010. Spectral Methods for Uncertainty Quantification. New York: Springer.

Lestoille, N., C. Soize, and C. Funfschilling 2015. Sensitivity of train stochastic dynamics to long-time evolution of track irregularities. Vehicle System Dynamics, submitted.

Lestoille, N., C. Soize, G. Perrin, and C. Funfschilling 2014. Long time evolution of train dynamics with respect to track geometry. In The Second International Conference on Railway Technology: Research, Development and Maintenance.

Mizuno, Y., Y. Fujino, K. Kataoka, and Y. Matsumoto 2008. Development of a Mobile Sensing Unit and its prototype implementation. Tsinghua Science \& Technology, 13:223-227.

Perrin, G., C. Soize, D. Duhamel, and C. Funfschilling 2012. Identification of polynomial chaos representations in high dimension from a set of realizations. SIAM Journal on Scientific Computing, 34(6):A2917-A2945.

Perrin, G., C. Soize, D. Duhamel, and C. Funfschilling 2013. Track irregularities stochastic modeling. Probabilistic Engineering Mechanics, 34:123-130.

Perrin, G., C. Soize, D. Duhamel, and C. Funfschilling 2015. Quantification of the influence of the track geometry variability on the train dynamics. Mechanical Systems and Signal Processing, 60:945-957.

Priestley, M. B. 1981. Spectral Analysis and Time Series. Academic Press, New York.

Priestley, M. B. 1988. Non-linear and non-stationary time series analysis. London: Academic Press.

Soize, C. 2012. Stochastic Models of Uncertainties in Computational Mechanics. Reston, VA, USA: American Society of Civil Engineers, Reston.

UIC 2009. Testing and approval of railway vehicles from the point of view of their dynamic behaviour - Safety - Track fatigue - Running behaviour. Leaflet 518. 